\title{
A Class of Solutions of Einstein's Equations which Admit a 3-Parameter Group of Isometries
}

\author{
J. M. Foyster and C. B. G. MCIntosh \\ Department of Mathematics, Monash University, Clayton, Vic., Australia
}

Received April 3, 1972

\begin{abstract}
The Plebaǹski and Stachel and Goenner and Stachel lists of metrics which are solutions of Einstein's field equations, have two double eigenvalues and admit 3-parameter groups of isometries with 2-dimensional spacelike orbits are completed by the addition of metrics which result from the use of a more general metric form.
\end{abstract}

\section{Introduction}

Plebaǹski and Stachel [1] state that they have carried out a complete classification of spherically-symmetric metrics whose Einstein tensors have two double eigenvalues. Goenner and Stachel [4] extend Ref. [1] to include the cases in which the two-curvature of the spacelike orbit may be zero or negative. However, as was pointed out by Takeno and Kitamura [2] and Goenner [3] the general form of the metric used by Plebaǹski and Stachel and Goenner and Stachel

where

$$
d s^{2}=e^{v}\left(d x^{0}\right)^{2}-e^{\lambda} d r^{2}-r^{2} d \omega^{2}
$$

$$
d \omega^{2}=d \theta^{2}+\Sigma^{2} d \phi^{2}
$$

and also where $\Sigma= \begin{cases}\sin \theta & \text { for positive two-curvature } \\ \sinh \theta & \text { for negative two-curvature } \\ 1 & \text { for zero two-curvature }\end{cases}$

in fact excludes one class of metrics. Here $\lambda=\lambda\left(x^{0}, r\right)$ and $v=v\left(x^{0}, r\right)$.

Goenner [3] extends Ref. [4] to include the class which had previously been omitted without, however, specifying the additional metrics involved. These metrics are listed in this paper.

From Goenner [3] the general form of a metric which admits a three parameter group of isometries with two-dimensional spacelike orbits (i.e. $\left.G_{3}(2, s)\right)$ is

$$
d s^{2}=2 G(u, v) d u d v-M^{2}(u, v)\left(d \theta^{2}+\Sigma^{2} d \phi^{2}\right) .
$$


The condition that two double eigenvalues exists is (see Appendix A)

$$
G M_{, v v}-G_{, v} M_{, v}=0
$$

such that either

$$
M_{, v}=A G,
$$

where $A$ is a function of $u$ only, or

$$
M_{, v}=0 .
$$

The case (5) is the one omitted by Plebaǹski and Stachel and Goenner and Stachel.

In the case (4) $A$ can be made one by a coordinate transformation. Then (2) becomes

$$
d s^{2}=2 M_{, v} d u d v-M^{2}(u, v) d \omega^{2} .
$$

The case examined by Plebanski and Stachel is the one for which the surfaces $M=$ constant are spacelike.

We put

$$
M(u, v)=r, \quad-2 M_{, u}=F
$$

and (6) becomes

where $F>0$.

$$
d s^{2}=F(u, r) d u^{2}+2 d u d r-r^{2} d \omega^{2}
$$

A complete list of metrics of the form (8) and of Petrov-Plebaǹski types $[2 N-2 S]_{[2-1]}(A, B),[2 T-2 S]_{[1-1]}(A, B),[4 N]_{[2]}(A, B)$ and $[4 T]_{[1]}(A, B)$ is given in Ref. [1] for $\lambda=-1$ and in Table V of Ref. [4] for $\lambda=0, \pm 1$. Here

$$
\lambda=\left[d^{2} \Sigma(\theta) / d \theta^{2}\right] / \Sigma .
$$

In the above description of the metric types, $A=0$ for zero curvature invariant $R$, and $A=R$ otherwise, $B$ is the Petrov type of the Weyl tensor (types $\mathrm{D}$ and $\mathrm{O}$ only for metric (2)) and the other symbols give the various Petrov-Plebaǹski types of the Ricci tensor (see Refs. [1] and [4]).

It is to be noted that the constant $e^{2}$ which appears in two of the Goenner and Stachel solutions can be negative or positive, the positive $e^{2}$ having been chosen for the physical interpretation.

It would appear from Part 4 of Ref. [1] that there are no sphericallysymmetric metrics with $(A, B)=(0,0)$ other than the flat solution $[4 T]_{[1]}(0,0)$. A similar conclusion for metrics admitting $G_{3}(2, s)$ would seem to follow from Table V of Ref. [4]. Appropriate metrics of these classes do in fact exist and take the form of metric (2) with (5) holding. 


\section{Classification of the Metrics of Case (5)}

After making coordinate transformations it is clear that the metrics with (5) holding have the form of either

or

$$
\begin{gathered}
d s^{2}=2 G(u, v) d u d v-d \omega^{2} \\
d s^{2}=2 G(u, v) d u d v-u^{2} d \omega^{2} .
\end{gathered}
$$

All such metrics have two double eigenvalues and the Ricci scalar is zero if and only if the Petrov type of the Weyl tensor is 0 , i.e. $(A, B)$ $=(R, D)$ or $(0,0)$. The classification of these metrics follows the method discussed in the Appendix.

The metrics of the form (10) are:

(i) $[4 T]_{[1]}(0,0)$, flat space,

$$
d s^{2}=2 U V d u d v-d \omega^{2}
$$

where $\lambda=0$ (i.e. $d \omega^{2}=d x^{2}+d y^{2}$ ) and $U$ and $V$ are functions of $u$ and $v$ respectively.

(ii) $[2 T-2 S]_{[1-1]}(0,0)$

$$
d s^{2}=\frac{4 U^{\prime} V^{\prime}}{\lambda(U+V)^{2}} d u d v-d \omega^{2}
$$

where $\lambda \neq 0$.

Here $G=\frac{2 U^{\prime} V^{\prime}}{\lambda(U+V)^{2}}$ is the solution of Liouville's equation

$$
\left.\frac{G_{, u}}{G}\right)_{, v}=\lambda G
$$

(see Goursat [5]).

(iii) $[4 T]_{[1]}(R, D)$

$$
d s^{2}=\frac{-4 U^{\prime} V^{\prime}}{\lambda(U+V)^{2}} d u d v-d \omega^{2} .
$$

(iv) $[2 T-2 S]_{[1-1]}(R, D)$

where

$$
d s^{2}=2 G(u, v) d u d v-d \omega^{2}
$$

(a) if $\lambda=0, G(u, v)$ is not the product of a function of $u$ only and a function of $v$ only:

(b) if $\lambda \neq 0, G(u, v)$ is not a solution of Liouville's Eq. (14). 
The metrics of the form (11) are

(v) $[4 T]_{[1]}(0,0)$, flat space,

$$
d s^{2}=2 V d u d v-u^{2}\left(d x^{2}+d y^{2}\right) .
$$

(vi) $[2 T-2 S]_{[1]}(R, D)$

with $\lambda \neq 0$.

$$
d s^{2}=2 V d u d v-u^{2} d \omega^{2}
$$

(vii) $[4 N]_{[2]}(0,0)$

with $U^{\prime} \neq 0$.

$$
d s^{2}=2 U V d u d v-u^{2}\left(d x^{2}+d y^{2}\right)
$$

(viii) $[4 N]_{[2]}(R, D)$

$$
d s^{2}=\frac{-4 U^{\prime} V^{\prime} u^{2}}{\lambda(U+V)^{2}} d u d v-u^{2} d \omega^{2} .
$$

(ix) $[2 N-2 S]_{[2-1]}(0,0)$

$$
d s^{2}=\frac{4 U^{\prime} V^{\prime} u^{2}}{\lambda(U+V)^{2}} d u d v-u^{2} d \omega^{2} .
$$

For metrics (viii) and (ix) we also have

$$
\left\{U^{\prime \prime} V^{\prime}(U+V)-U^{\prime 2} V^{\prime}\right\} u+2 U^{\prime} \neq 0 .
$$

(x) $[2 N-2 S]_{[2-1]}(R, D)$

$$
d s^{2}=2 G(u, v) d u d v-u^{2} d \omega^{2}
$$

where $G$ does not take the forms required for (vii)-(ix). Discussion of simplification of some of these metrics is to be found in Appendix B.

\section{Appendix A}

The metric (2) can be written in terms of a pseudoorthonormal tetrad (see Newman and Penrose [6]) as

$$
\begin{aligned}
l^{\mu} & =\left(0, \frac{1}{G}, 0,0\right) \\
n^{\mu} & =(1,0,0,0) \\
m^{\mu} & =-\frac{1}{M \sqrt{2}}\left(0,0,1, \frac{i}{\Sigma}\right) .
\end{aligned}
$$


For this tetrad we have the non-zero Newman-Penrose quantities

$$
\begin{aligned}
\Psi_{2} & =\frac{M_{, u v}}{M G}-2 \Lambda, \\
\Lambda & =\frac{1}{12}\left(\frac{G_{, u v}}{G^{2}}-\frac{G_{, u} G_{\cdot v}}{G^{3}}-\frac{\lambda}{M^{2}}\right)+\frac{1}{3}\left(\frac{M_{, u v}}{M G}+\frac{M_{, u} M_{, v}}{2 G M^{2}}\right), \\
\Phi_{00} & =\frac{M_{. v} G_{\cdot v}}{M G^{3}}-\frac{M_{, v v}}{M G^{2}}, \\
\Phi_{22} & =\frac{M_{. u} G_{, u}}{M G}-\frac{M_{, u u}}{M}, \\
\Phi_{11} & =\frac{1}{4}\left(\frac{G_{, u} G_{, v}}{G^{3}}-\frac{G_{. u v}}{G^{2}}-\frac{\lambda}{M^{2}}\right)+\frac{M_{. u} M_{\cdot v}}{2 G M^{2}}
\end{aligned}
$$

where $\Psi_{2}$ is related to the Weyl tensor, $\Lambda=R / 24, \Phi_{A B}$ are related to the Ricci tensor and $\lambda$ is defined by (9) in this paper.

From a comparison of Ludwig and Scanlan [7] and Ref. [1] it follows that the metric has two double eigenvalues if

$$
\Phi_{00}=0
$$

(or $\Phi_{22}=0$ ) which leads to Eq. (3).

Then the metric is of type

$$
\begin{array}{llll}
{[2 N-2 S]_{[2-1]}} & \text { when } & \Phi_{11}, \Phi_{22} & \text { are non-zero } \\
{[2 T-2 S]_{[1-1]}} & \text { when } & \Phi_{11} & \text { is non-zero } \\
{[4 N]_{[2]}} & \text { when } & \Phi_{22} & \text { is non-zero } \\
{[4 T]_{[1]}} & \text { when } & \text { all } \Phi_{A B} & \text { are zero. }
\end{array}
$$

and $(0,0)$ implies $\Psi_{2}=0$ and $\Lambda=0$.

The above classification applied to metrics (10) and (11) gives the metrics (i)-(x).

\section{Appendix B}

In metrics (v), (vi) and (vii) $V$ can be made unity by a coordinate transformation. The transformation

$$
W=v+\frac{1}{2} x^{2} u+\frac{1}{2} y^{2} u, \quad U=u, \quad X=x u, \quad Y=y u
$$

takes metric $(\mathrm{v})$ into the Minkowski metric

$$
d s^{2}=2 d U d W-d X^{2}-d Y^{2} .
$$


Metric (vii) is a purely non-gravitational plane wave metric. Penrose [8] has discussed such a metric in the case of a purely electromagnetic wave but it should be noted that the wave does not have to be a null electromagnetic one but may be a wave of some other energy form such as a scalar wave (see Lun and McIntosh [9]).

Metrics (ii), (iii), (viii) and (ix) can also be simplified. For example (iii) is immediately

$$
d s^{2}=\frac{4 d U d V}{\lambda(U+V)^{2}}-d \omega^{2} .
$$

Acknowledgement. We thank Dr. E. D. Fackerell for helpful discussions on the work of this paper.

\section{References}

1. Plebaǹski, J., Stachel, J.: J. Math. Phys. 9, 269 (1968).

2. Takeno, H., Kitamura, S.: On the Einstein tensors of spherically symmetric space-times. Hiroshima University RRK 69-13 (1969).

3. Goenner, H.: Commun. math. Phys. 16, 34 (1970).

4. - Stachel, J.: J. Math. Phys. 11, 3358 (1970).

5. Goursat,E.: A course in mathematical analysis. Vol. III, Part 1, p. 71. New York: Dover Publ. 1964.

6. Newman, E. T., Penrose, R.: J. Math. Phys. 3, 566 (1962).

7. Ludwig, G., Scanlan, G.: Commun. math. Phys. 20, 291 (1971).

8. Penrose, R.: Rev. Mod. Phys. 37, 215 (1965).

9. Lun, A. W.C., McIntosh, C. B. G.: Preprint (1972).

J. M. Foyster

C.B.G. McIntosh

Department of Mathematics

Monash University

Clayton, Victoria, 3168

Australia 